- 研究报告・

\title{
不同进化类型大肠杆菌的适合度频率依赖性
}

\author{
倪川 朱璧如 张大勇* \\ (北京师范大学生物多样性与生态工程教育部重点实验室, 北京 100875)
}

\begin{abstract}
摘要: 进化实验中使用竞争实验测量两个物种或基因型的相对适合度时，通常假定两者的适合度差异是恒定的。 然而竞争双方的相互作用可能导致适合度差异受到初始相对频率的影响。本研究通过测量两种进化株系的大肠杆 菌(Escherichia coli) 在不同初始相对频率下的相对适合度, 分析了初始相对频率对相对适合度的影响。结果发现高 氮进化株系相对低氮进化株系的相对适合度随其初始相对频率的减小而增加, 表现出显著的负频率依赖。两种株 系在培养高氮株系的滤出液中均没有显著的生长, 在培养低氮株系的滤出液中均有生长, 但高氮株系的细胞加倍 次数是低氮株系的 3 倍, 说明导致适合度出现负频率依赖的原因可能是低氮株系的竞争能力较低, 不能完全利用 培养基中的资源; 也可能是低氮株系的代谢产物能被高氮株系利用, 产生互养作用。不同的相互作用可能导致不 同的初始相对频率与相对适合度间的关系, 因此, 为了更准确地衡量适合度, 进化实验中需要考虑初始相对频率 对相对适合度的影响。
\end{abstract}

关键词: 竞争实验; 初始相对频率; 互养作用; 他感作用

\section{Frequency dependent fitness in different evolved Escherichia coli lines}

\author{
Chuan Ni, Biru Zhu, Dayong Zhang*
}

Key Laboratory for Biodiversity Science and Ecological Engineering of Ministry of Education, Beijing Normal University, Beijing 100875

\begin{abstract}
Differences in fitness between two species or genotypes is usually assumed to be constant when competition experiments are used to measure relative fitness in evolutionary experiments. However, interactions between competitors may lead to frequency-dependence in fitness. We measured the relative fitness of two types of evolved lines of Escherichia coli under different initial relative frequencies to analyze the effects of initial relative frequency on relative fitness. Competed with the low nitrogen evolved lines, the high nitrogen evolved lines displayed increased relative fitness with decreased initial relative frequency, which suggests negative frequency dependence. Both types did not grow in the filtrate from high nitrogen evolved lines, but grew in the filtrate from low nitrogen evolved lines. However, the number of cell doublings of the high nitrogen evolved lines was three times higher than that of the low nitrogen evolved lines. One probable explanation for the negative frequency dependent fitness was that the low nitrogen evolved lines had weaker resource competitive ability and could not sufficiently use resources. Another explanation was that the high nitrogen evolved lines could use some metabolites produced by the low nitrogen evolved lines, which suggests the existence of cross-feeding interaction. Different interactions may lead to different relationships between relative fitness and initial relative frequency. Therefore, we need to account for the effects of initial relative frequency on relative fitness to more accurately measure fitness in evolutionary experiments.
\end{abstract}

Key words: competition experiment; initial relative frequency; cross-feeding interaction; allelopathic interaction

竞争实验是进化生物学中测量两个物种或基 因型相对适合度的常用方法。该方法通过测量竞争
双方的相对频率经过一段时间后的变化来衡量相 对适合度, 相对频率增加的一方具有较高的适合

收稿日期: 2016-05-30; 接受日期: 2016-06-13

基金项目: 国家自然科学基金(31030014)

* 通讯作者 Author for correspondence. E-mail: zhangdy@bnu.edu.cn 
度。通常这种方法假定两者之间的适合度差异是恒 定的(Lenski, 1988; Lenski et al, 1991)。然而生态学 中很多竞争实验的结果表明两个物种或基因型的 竞争结果是频率依赖的(Ayala，1971; Debenedictis, 1977; Harpole \& Suding, 2007; Kerswell \& Burd, 2012), 因此基于竞争实验测量的相对适合度也可 能是频率依赖的, 即相对适合度会随初始相对频率 变化而变化。这种频率依赖性的产生可能与竞争双 方的相互作用例如他感作用(allelopathic interaction) 和互养作用(cross-feeding interaction)有关。

他感作用是指竞争双方中一方会产生有毒物 质而抑制另一方的生长, 甚至杀死另一方。如果产 毒的一方相对频率较高, 产生的有毒物质较多, 对 竞争者的抑制作用也越强, 这就会导致产毒者的相 对适合度随着其初始相对频率的增加而增大。Greig 和Travisano (2008)研究发现, 当产毒酵母的初始频 率较低时, 其相对适合度低于不产毒的酵母; 但随 着产毒酵母的初始频率增加, 其相对适合度也增 加。因此他感作用可能导致正频率依赖选择 (positive frequency dependent selection), 加速稀少 物种或基因型的灭绝, 降低多样性(Chao \& Levin, 1981)。不过最近的一些理论和实验研究表明, 当种 群呈斑块状分布在异质环境中时, 正频率依赖也可 能维持多样性(Molofsky et al, 2001; Molofsky \& Bever, 2002; Rendueles et al, 2015)。

与此相反, 互养作用是指竞争双方中的一方产 生的物质能被另一方利用, 从而导致能利用对方产 物的一方相对适合度大于产生可利用物质的一方。 这时能利用对方产物的一方的相对适合度将会随 着自身初始频率的降低而增大, 因为这时它们可利 用的资源更多。Ribeck和Lenski (2015)发现两种存 在互养作用的大肠杆菌实验体系中相对适合度均 随着初始频率的增加而降低。因此互养作用可能导 致负频率依赖选择 (negative frequency dependent selection), 从而促进物种共存 (Pfeiffer \& Bonhoeffer, 2004; Estrela \& Gudelj, 2010)。

由此可见，当两个竞争者间的相互作用不同 时, 相对适合度随初始相对频率变化的情况也不 同。所以进化生物学的研究应该充分考虑不同初始 相对频率对相对适合度的影响, 这将有助于我们研 究频率依赖选择, 理解不同相互作用在频率依赖选 择中的作用及其对生物多样性的影响。但是目前的
进化实验研究很少考虑相对适合度的变化(Ribeck \& Lenski, 2015)。因此, 本研究测量了两种不同进化 类型的大肠杆菌 (Escherichia coli) 在不同初始相对 频率情况下的相对适合度, 检测初始相对频率是否 会影响相对适合度; 通过分析相对适合度随初始相 对频率变化的模式, 探讨两种进化类型的大肠杆菌 间可能存在的相互作用。

\section{1 材料与方法}

\section{1 实验菌株}

本研究中使用的两种进化类型的大肠杆菌株 系是由K-12 GM4792株系分别在高、低两种氮资源 环境下进化 1,300 代后得到的, 通过调节培养基中 $\mathrm{NH}_{4} \mathrm{Cl}$ 的含量, 最终使高氮资源环境中氮元素含量 约为 $654 \mathrm{mg} / \mathrm{L}$, 低氮资源环境中氮元素含量约为 $4.36 \mathrm{mg} / \mathrm{L} 。 1,300$ 代后, 进化株系的大肠杆菌在各自 环境中的适合度均显著大于祖先株系, 说明它们均 对自己生长的环境产生了适应性进化(倪川, 2011)。 高氮环境中进化的株系标记为 $\mathrm{N}+$, 低氮环境中进 化的株系标记为 $\mathrm{N}-$, 两种进化株系分别有 10 个重 复的种群。这 10 个重复种群中有 5 个种群带有 $L a c+$ 标记, 可以分解乳糖, 另外 5 个种群带有 $L a c-$ 标记, 不能分解乳糖。两种标记的大肠杆菌能在含有X-gal 的LB培养基上分别形成蓝色和白色菌落, 因此可 以用含有不同 $L a c$ 标记的两种进化株系进行竞争实 验, 测量相对适合度。

\section{2 相对适合度测量}

所有的竞争实验都是在高氮资源的MOPS培养 基中进行, 培养基具体配方参见倪川(2011)。竞争实 验之前所有的竞争株系都要单独在该培养基中驯 化培养 $24 \mathrm{~h}$ 。将 $\mathrm{N}+$ 株系和 $\mathrm{N}-$ 株系的 10 个重复种群随 机配对, 要求每个竞争对含有不同的 $L a c$ 标记, 然 后得到 10 个竞争对。分别测量每一个竞争对在 5 种 不同初始相对频率下 $\mathrm{N}+$ 株系对 $\mathrm{N}-$ 株系的相对适合 度。通过调整竞争双方混合的比例使得 $\mathrm{N}+$ 株系的初 始相对频率分别为: $0.01 、 0.1 、 0.5 、 0.9 、 0.99$ 。两 个竞争者驯化培养后按照设置的初始频率取相应 体积的菌液混匀, 将混合菌液以 100 倍的稀释倍数 接入含有 $160 \mu \mathrm{L}$ 新鲜培养基的 96 孔微孔板中, $37^{\circ} \mathrm{C}$, $200 \mathrm{rpm}$ 振荡培养 $24 \mathrm{~h}$; 同时取部分混合菌液稀释到 合适的浓度后, 取 $100 \mu \mathrm{L}$ 涂布到含有X-gal的LB培 养基平板上, $37^{\circ} \mathrm{C}$ 静止培养过夜, 通过计数平板上 
蓝色和白色菌落的个数来计算初始时刻两种进化 株系的种群密度 $\left(N_{+}(0)\right.$ 和 $\left.N_{-}(0)\right)$ 。前述混合菌液培养 $24 \mathrm{~h}$ 后, 再次使用相同的方法测量两种进化株系的 种群密度 $\left(N_{+}(1)\right.$ 和 $\left.N_{-}(1)\right)$, 按照Lenski等(1991)的方 法计算 $\mathrm{N}+$ 株系对 $\mathrm{N}-$ 株系的相对适合度： $w=\frac{\ln \left(N_{+}(1) / \ln \left(N_{+}(0)\right)\right.}{\ln \left(N_{-}(1) / \ln \left(N_{-}(0)\right)\right.} 。 w>1$ 说明 $\mathrm{N}+$ 株系的适合度 大于 $\mathrm{N}-$ 株系, $w=1$ 说明 $\mathrm{N}+$ 株系的适合度等于 $\mathrm{N}-$ 株 系, $w<1$ 说明 $\mathrm{N}+$ 株系的适合度小于 $\mathrm{N}-$ 株系。每个竞 争对在每种初始频率条件下重复测量两遍相对适 合度，然后取平均值。

\section{3 滤出液中生长情况的测量}

为了进一步分析竞争双方产生的物质对竞争 者的影响, 我们测量了两种进化株系分别在自己和 竞争对手生长后的滤出液中的生长情况。大肠杆菌 的繁殖方式是无性的细胞分裂，因此可以用相同时 间内细胞加倍的次数来衡量生长情况。将 $24 \mathrm{~h}$ 驯化 培养后的菌液按 100 倍稀释倍数接入 $160 \mu \mathrm{L}$ 和 $5 \mathrm{~mL}$ 新鲜培养基中, $37^{\circ} \mathrm{C}, 200 \mathrm{rpm}$ 振荡培养 $24 \mathrm{~h}$ 。之后将 $5 \mathrm{~mL}$ 菌液用 $0.2 \mu \mathrm{m}$ 无菌滤器过滤, 除掉其中的细 菌, 得到滤出液。 $\mathrm{N}+$ 株系产生的滤出液标记为 $\mathrm{N}+\mathrm{F}$, $\mathrm{N}-$ 株系产生的滤出液标记为 $\mathrm{N}-\mathrm{F}$ 。按照相对适合度 测量中设置的竞争对, 从培养了 $24 \mathrm{~h}$ 的 $160 \mu \mathrm{L}$ 菌液 中分别取出 $1.6 \mu \mathrm{L}$ 的菌液接入 $160 \mu \mathrm{L}$ 自己的滤出液 和竞争对手的滤出液中, $37^{\circ} \mathrm{C}, 200 \mathrm{rpm}$ 振荡培养 24 $\mathrm{h}$, 同时使用前述的涂板方法测量初始时的种群密 度 $\left(N_{0}\right)$ 。2 $4 \mathrm{~h}$ 后, 再次测量种群密度 $\left(N_{1}\right)$ 。计算每个 种群在 $24 \mathrm{~h}$ 内细胞加倍的次数: $D=\ln \left(N_{1} / N_{0}\right) / \ln 2$ 。 $D>0$ 说明种群数量增加, $D=0$ 说明种群数量不变, $D<0$ 说明种群数量减小。每个种群只测量 1 次。

\section{4 数据分析}

所有的数据分析都使用 R 软件 3.1.3 (R Core Team, 2015)。首先用单样本 $t$ 检验分别检验每一种初 始相对频率情况下, $\mathrm{N}+$ 株系相比于 $\mathrm{N}-$ 株系的相对 适合度是否显著偏离 1 。然后计算每一种初始相对 频率下相对适合度的平均值, 对平均相对适合度和 初始相对频率进行回归分析，分析相对适合度随初 始相对频率的变化情况。另外我们把初始相对频率 作为因子, 用单因素方差分析(one-way ANOVA)检 验不同初始相对频率下的相对适合度是否有显著 差异。用单样本 $t$ 检验分别检验 $\mathrm{N}+$ 和 $\mathrm{N}-$ 两种进化株 系在 $\mathrm{N}+\mathrm{F}$ 和 $\mathrm{N}-\mathrm{F}$ 两种滤出液中的生长率是否显著偏 离于 0 。用两个独立样本 $t$ 检验分别检验两种不同滤 出液中, 两种进化株系的生长率是否有显著差异。

\section{2 结果}

在实验设置的 5 种初始相对频率下, $\mathrm{N}+$ 株系相 对于 $\mathrm{N}-$ 株系的相对适合度均显著大于 1 (表1)。说明 在各种初始相对频率下 $\mathrm{N}+$ 株系的适合度均大于 $\mathrm{N}-$ 株系。但是 $\mathrm{N}+$ 株系的相对适合度随着它的初始相对 频率的增加而减小，回归分析显示 $\mathrm{N}+$ 株系的相对 适合度与其初始相对频率有显著的非线性负相关 关系(图1A)。说明 $\mathrm{N}+$ 株系在初始相对频率较低的时 候具有较高的适合度，存在负频率依赖。单因素方 差分析结果表明初始相对频率对相对适合度有显 著影响 $(F=22.08, \mathrm{df}=4, P<0.001)$, 多重比较 (Tukey HSD检验)结果发现初始相对频率主要在较 小的时候对相对适合度有显著影响，当初始频率大 于 0.5 以后, $0.5 、 0.9$ 和 0.99 三种初始相对频率下的相 对适合度之间并没有显著差异(表1)。

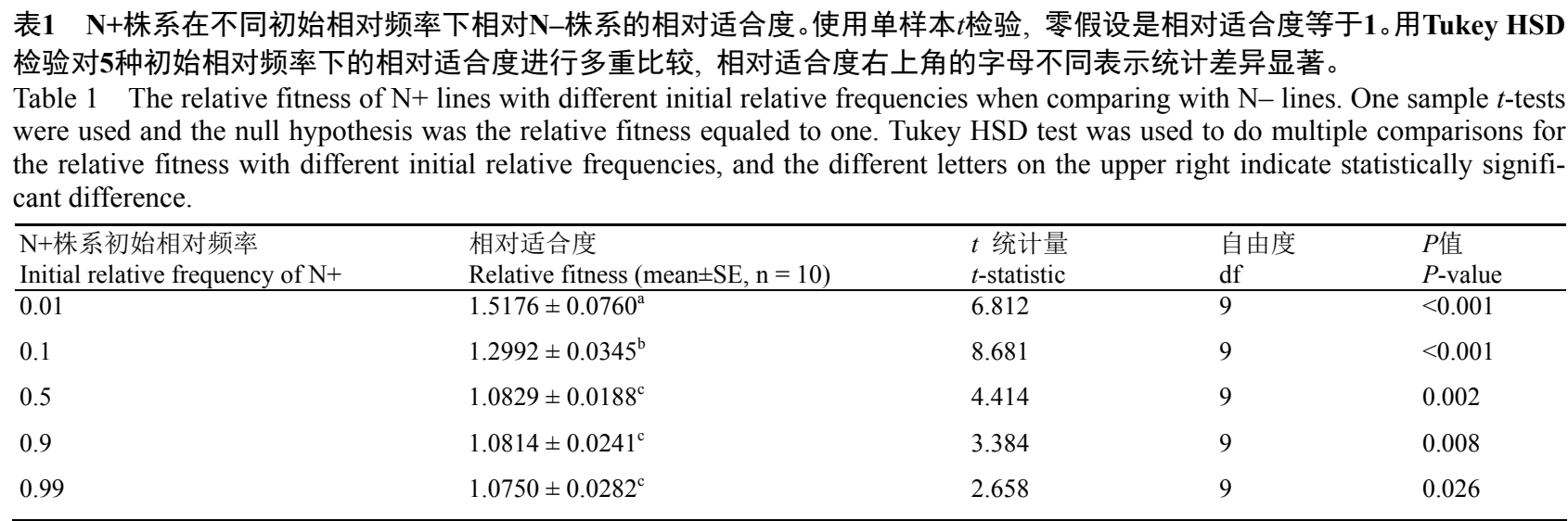



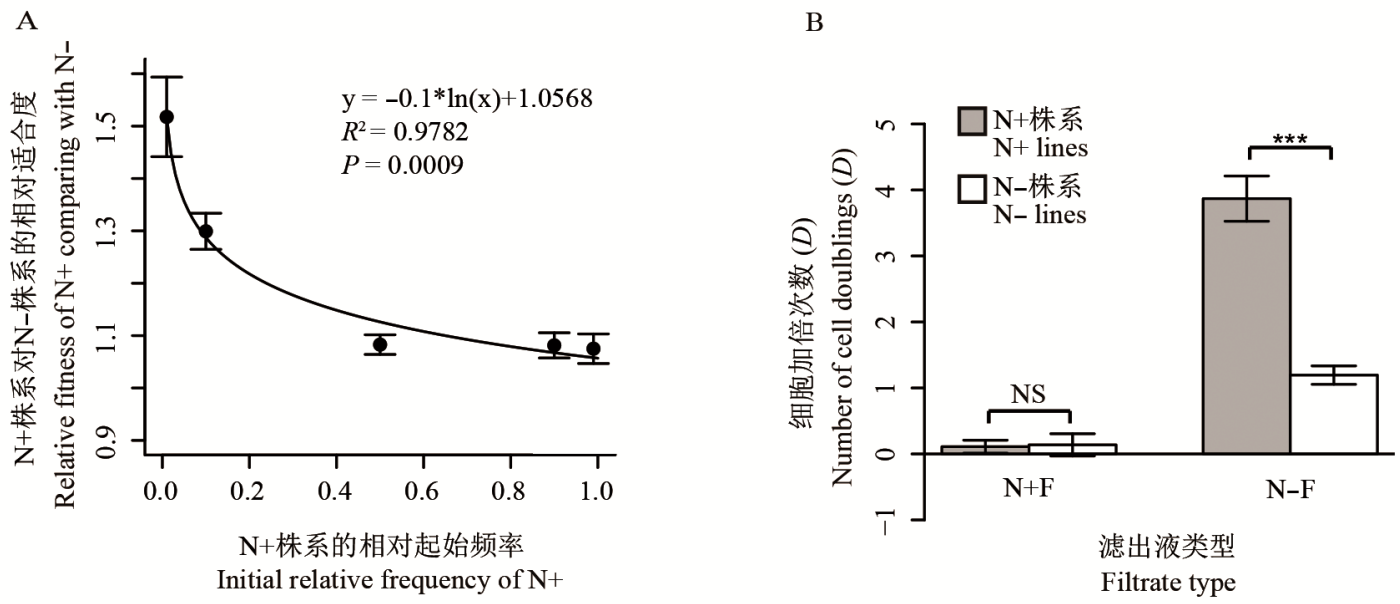

图1 N+株系的相对适合度与其初始相对频率的相关性(A)及两种进化株系的大肠杆菌在两种滤出液中的 $24 \mathrm{~h}$ 内细胞加倍次 数(B)。(A)图中黑点表示每种初始相对频率下 $\mathrm{N}+$ 株系相对于 $\mathrm{N}-$ 株系的相对适合度的平均值, 误差线表示标准误 $(n=10)$ 。图 中曲线拟合的是平均相对适合度与初始相对频率的关系，右上角显示了拟合的方程和统计显著性。(B)图中数据显示了均值 土标准误 $(n=10)$ 。 $N+F$ 和 $N-F$ 是分别是 $N+$ 株系和 $N-$ 株系培养 $24 \mathrm{~h}$ 后的滤出液。*** $P<0.001, N S$ 表示 $P>0.05$ (两个独立样本 $t$ 检验)。

Fig. 1 The relationship between relative fitness and initial relative frequency of $\mathrm{N}+$ lines $(\mathrm{A})$, and the number of cell doublings $(D)$ of two types of evolved Escherichia coli lines in two different filtrates (B). (A) The black dots show the mean relative fitness of N+ lines comparing with $\mathrm{N}$ - lines, and the error bars show SE $(\mathrm{n}=10)$. The curve indicates the regression model, whose equation and statistical significance are showed upper right. (B) Data show mean $\pm \mathrm{SE}(\mathrm{n}=10)$. N+F and N-F indicate filtrate prepared from 24 $\mathrm{h}$ cultures of $\mathrm{N}+$ lines and $\mathrm{N}-$ lines, respectively. ${ }^{* * *} P<0.001$, and NS indicates $P>0.05$ (two independent sample $t$-tests).

两种进化株系在 $\mathrm{N}+\mathrm{F}$ 滤出液中均没有生长, $\mathrm{N}+$ 株系的细胞加倍次数为 $0.110 \pm 0.098$ (平均值 \pm 标准 误, $\mathrm{n}=10), \mathrm{N}-$ 株系的细胞加倍次数为 $0.137 \pm 0.169$, 单样本 $t$ 检验显示两者均不显著偏离 $0(\mathrm{~N}+$ 株系: $t=$ $1.1266, \mathrm{df}=9, P=0.289 ; \mathrm{N}-$ 株系: $t=0.8149, \mathrm{df}=9$, $P=0.436)$, 并且两者间没有显著的差别 $(t=-0.138$, $\mathrm{df}=14.467, P=0.892)($ 图1B)。说明培养 $\mathrm{N}+$ 株系 24 $\mathrm{h}$ 以后, 培养基中可利用的资源已经耗尽, 两种进 化株系的种群数量保持不变。两种进化株系在 N-F 滤出液中均有生长, $\mathrm{N}+$ 株系的细胞加倍次数为 $3.8691 \pm 0.3430, \mathrm{~N}-$ 株系的细胞加倍次数为 $1.1948 \pm$ 0.1398 , 单样本 $t$ 检验的结果显示两者均显著大于 1 $(\mathrm{N}+$ 株系: $t=11.279, \mathrm{df}=9, P<0.001 ; \mathrm{N}-$ 株系: $t=$ $8.5439, \mathrm{df}=9, P<0.001)$ 。说明培养 $\mathrm{N}-$ 株系 $24 \mathrm{~h}$ 以 后, 培养基中还有可以被利用的资源。虽然两种进 化株系在 $\mathrm{N}-\mathrm{F}$ 滤出液中均有生长, 但是 $\mathrm{N}+$ 株系的生 长显著大于 $\mathrm{N}-$ 株系 $(t=7.219, \mathrm{df}=11.911, P<$ 0.001)(图1B)。

\section{3 讨论}

本研究测量了两种进化类型的大肠杆菌在不 同初始相对频率下的相对适合度, 发现 $\mathrm{N}+$ 株系相
对 $\mathrm{N}-$ 株系的相对适合度随着 $\mathrm{N}+$ 株系初始相对频率 的减小而增加, 表现为负频率依赖。这种负频率依 赖可能与两种进化株系资源竞争能力的差异有关。 生物对有限资源的竞争能力可以用其降低环境中 有限资源含量的能力表示，即竞争能力越强，越能 充分利用环境中的有限资源 (Tilman \& Wedin, 1991)。滤出液的结果显示两种大肠杆菌株系在 N+F 滤出液中均没有显著的生长, 但在 $\mathrm{N}-\mathrm{F}$ 滤出液中均 能生长(图1B), 说明 $\mathrm{N}+$ 株系在 $24 \mathrm{~h}$ 后就已经将培养 基中的资源消耗殆尽，而 $\mathrm{N}-$ 株系则不能完全利用 培养基中的资源。这表明 $\mathrm{N}+$ 株系竞争资源的能力大 于 $\mathrm{N}-$ 株系。因此当 $\mathrm{N}-$ 株系相对频率增加时, 由于 $\mathrm{N}-$ 株系不能充分利用资源, $\mathrm{N}+$ 株系能利用的资源相对 增加，有利于种群增长，其相对适合度增加。

另外，相对适合度的负频率依赖也可能暗示了 这两种进化类型的大肠杆菌间存在互养作用。在资 源贫乏的环境中, 生物可能通过快速吸收有限的资 源来提高自己的适合度，但资源的吸收和利用常常 存在权衡关系，即快速的资源吸收可能导致低效的 资源利用。例如在葡萄糖受限环境中进化的大肠杆 菌虽然提高了吸收葡萄糖分子的能力，但是不能充 分代谢葡萄糖分子, 因而产生部分降解的代谢产 
物, 这些代谢产物可能被其他株系利用, 从而形成 互养关系(Helling et al, 1987; Rosenzweig et al, 1994; Turner et al, 1996; Treves et al, 1998; Rozen \& Lenski, 2000)。本研究中使用的 $\mathrm{N}-$ 株系是在极度缺 氮的环境中进化的, 提高了吸收氮资源的速率(倪 川，2011)，因此有可能不能充分利用吸收的资源， 产生代谢不完全的产物, 这些产物能被 $\mathrm{N}+$ 株系利 用，从而形成互养作用。在培养 $\mathrm{N}-$ 株系 $24 \mathrm{~h}$ 后的滤 出液中, 虽然两种株系的大肠杆菌均有生长, 但是 $\mathrm{N}+$ 株系的生长率约是 $\mathrm{N}-$ 株系的 3 倍, 可能是因为 $\mathrm{N}-$ 株系无法彻底利用培养基中的资源, 也有可能 是 $\mathrm{N}-$ 株系分泌的某些代谢产物能被 $\mathrm{N}+$ 株系利用, 促进它们的生长。

虽然 $\mathrm{N}+$ 株系相对 $\mathrm{N}-$ 株系的相对适合度表现出 负频率依赖, 但 $\mathrm{N}+$ 株系的相对适合度始终显著大 于 $\mathrm{N}-$ 株系, 并且当 $\mathrm{N}+$ 株系的初始相对频率大于 0.5 以后, $\mathrm{N}+$ 株系相对 $\mathrm{N}-$ 株系的相对适合度基本保持 一致(图1A)。Ribeck和Lenski (2015)的模型预测, 如 果只存在互养作用, 那么相对适合度随初始相对频 率的变化应该是线性负相关, 如果不是线性, 说明 还存在其他的相互作用。在本研究中, 当 $\mathrm{N}+$ 株系初 始相对频率较高时, 互养作用的效用较小, 这可能 是由于他感作用导致了 $\mathrm{N}+$ 株系较高的适合度。但在 $\mathrm{N}+\mathrm{F}$ 滤出液中, 两种进化株系均没有显著的生长, 且两者的生长率并没有显著差异, 说明 $\mathrm{N}+$ 株系的 代谢产物并未对 $\mathrm{N}$-株系产生毒害作用。因此本研究 中使用的两种大肠杆菌株系间不太可能存在他感 作用。另一种可能的解释就是当 $\mathrm{N}+$ 株系的初始相对 频率大于 0.5 以后, 双方的相互作用主要是资源竞 争, 并且相对竞争能力不再随初始相对频率变化。

本研究中相对适合度表现出明显的频率依赖 性, 这一结果显然与进化实验中通常假定的恒定相 对适合度不符，但不能确定在各种不同的进化实验 系统中是否普遍存在这种频率依赖的相对适合度 现象。因为通常采用竞争实验测量相对适合度时使 用的都是 0.5 的初始相对频率, 很少有研究在多种 初始相对频率下测量相对适合度。因此有必要在不 同的实验系统中衡量初始相对频率对相对适合度 的影响。这样不仅能更加准确地测量相对适合度, 还能通过分析初始相对频率与相对适合度间的相 关关系研究不同进化类型间可能存在的相互作用。

\section{参考文献}

Ayala FJ (1971) Competition between species: frequency dependence. Science, 171, 820-824.

Chao L, Levin BR (1981) Structured habitats and the evolution of anticompetitor toxins in bacteria. Proceedings of the National Academy of Sciences, USA, 78, 6324-6328.

Debenedictis PA (1977) Meaning and measurement of frequency-dependent competition. Ecology, 58, 158-166.

Estrela S, Gudelj I (2010) Evolution of cooperative cross-feeding could be less challenging than originally thought. PLoS ONE, 5, e14121.

Greig D, Travisano M (2008) Density-dependent effects on allelopathic interactions in yeast. Evolution, 62, 521-527.

Harpole WS, Suding KN (2007) Frequency-dependence stabilizes competitive interactions among four annual plants. Ecology Letters, 10, 1164-1169.

Helling RB, Vargas CN, Adams J (1987) Evolution of Escherichia coli during growth in a constant environment. Genetics, 116, 349-358.

Kerswell KJ, Burd M (2012) Frequency-dependent and density-dependent larval competition between life-history strains of a fly, Lucilia cuprina. Ecological Entomology, 37, 109-116.

Lenski RE (1988) Experimental studies of pleiotropy and epistasis in Escherichia coli. I. Variation in competitive fitness among mutants resistant to virus T4. Evolution, 42, 425-432.

Lenski RE, Rose MR, Simpson SC, Tadler SC (1991) Long-term experimental evolution in Escherichia coli 1: adaptation and divergence during 2000 generations. The American Naturalist, 138, 1315-1341.

Molofsky J, Bever JD (2002) A novel theory to explain species diversity in landscapes: positive frequency dependence and habitat suitability. Proceedings of the Royal Society B: Biological Sciences, 269, 2389-2393.

Molofsky J, Bever JD, Antonovics J (2001) Coexistence under positive frequency dependence. Proceedings of the Royal Society B: Biological Sciences, 268, 273-277.

Ni C (2011) The Experimental Evolution of Escherichia coli in Nitrogen Limited Environment. PhD dissertation, Beijing Normal University, Beijing. (in Chinese with English abstract) [倪川 (2011) 大肠杆菌在缺氮环境下的实验进 化. 博士学位论文, 北京师范大学, 北京.]

Pfeiffer T, Bonhoeffer S (2004) Evolution of cross-feeding in microbial populations. The American Naturalist, 163, E126-E135.

R Core Team (2015) R: A Language and Environment for Statistical Computing. https://www.r-project.org/

Rendueles O, Amherd M, Velicer GJ (2015) Positively frequency-dependent interference competition maintains diversity and pervades a natural population of cooperative microbes. Current Biology, 25, 1673-1681. 
Ribeck N, Lenski RE (2015) Modeling and quantifying frequency-dependent fitness in microbial populations with cross-feeding interactions. Evolution, 69, 1313-1320.

Rosenzweig RF, Sharp RR, Treves DS, Adams J (1994) Microbial evolution in a simple unstructured environment: genetic differentiation in Escherichia coli. Genetics, 137, 903-917.

Rozen DE, Lenski RE (2000) Long-term experimental evolution in Escherichia coli VIII: dynamics of a balanced polymorphism. The American Naturalist, 155, 24-35.
Tilman D, Wedin D (1991) Plant traits and resource reduction for 5 grasses growing on a nitrogen gradient. Ecology, 72, 685-700.

Treves DS, Manning S, Adams J (1998) Repeated evolution of an acetate-crossfeeding polymorphism in long-term populations of Escherichia coli. Molecular Biology and Evolution, 15, 789-797.

Turner PE, Souza V, Lenski RE (1996) Tests of ecological mechanisms promoting the stable coexistence of two bacterial genotypes. Ecology, 77, 2119-2129.

(责任编委: 张全国 责任编辑: 时意专) 